\title{
Ultra-high-aspect Multilayer Zone Plates for even higher X-ray energies
}

\author{
Markus Osterhoff' ${ }^{1}$ Jakob Soltau ${ }^{1}$, Christian Eberl ${ }^{2}$, Hans-Ulrich Krebs ${ }^{2}$ \\ ${ }^{1}$ Institut für Röntgenphysik, ${ }^{2}$ Institut für Materialphysik \\ University of Göttingen, Friedrich-Hund-Platz 1, D-37077 Göttingen
}

\begin{abstract}
Penetration lengths in the millimetre range make hard $\mathrm{x}$-rays above $60 \mathrm{keV}$ a well-suited tool for non-invasive probing of small specimens buried deep inside their surroundings, and enable studying individual components inside assembled, complex devices (solar cells, batteries etc.). The real-space resolution of typical imaging modalities like fluorescence mapping, scanning SAXS and WAXS depend on the available beam size. Although routine in the 5-25 keV regime [1-4], spot sizes below $50 \mathrm{~nm}$ are very challenging at $\mathrm{x}$-ray energies above $50 \mathrm{keV}$ : Compound refractive lenses lack in refractive power, the multilayer thickness of coated mirrors is bounded by interfacial diffusion, and lithographic Fresnel Zone Plates loose their efficiency in the two-digit keV regime.
\end{abstract}

Multilayer Laue Lenses and Multilayer Zone Plates (MZP) are promising candidates for high-keV focusing to small spot sizes; compared to Fresnel Zone Plates, the aspect ratio comparing outermost layer width ( focal spot size) to optical thickness (efficiency) is virtually unlimited by the fabrication. Using Pulsed Laser Deposition on a rotating wire (several millimetre long), we have fabricated an MZP with $10 \mathrm{~nm}$ outermost zone widths and optical thickness of $30 \mu \mathrm{m}$ (optimum phase shift at $60 \mathrm{keV}$ ), yielding an unprecedented ultra-high aspect ratio of 1:3000 (outermost zone width compared to optical thickness).

We present experimental results obtained at ESRF's high energy beamline ID31, where for the first time scanning experiments with real-space resolutions below $50 \mathrm{~nm}$ even at $\mathrm{x}$-ray energies ranging from $60 \mathrm{keV}$ to above $100 \mathrm{keV}$ have been achieved.

Keywords: Multilayer Zone Plates, high X-ray energies, Imaging, Focusing

\section{INTRODUCTION}

High X-ray energies beyond $30 \mathrm{keV}$ offer long penetration lengths into bulk material, so that even hidden and buried particles can be studied. Many composite materials are crystalline, with particle sizes in the sub- $\mu \mathrm{m}$ region; also, crystal domains within larger compounds of few hundred nm in size are common. So far, small structures can be studied $e x$-situ, i.e. as isolated or free-standing objects. But often, chemical and physical properties change when devices are assembled from individual components.

To efficiently investigate small particles at high spatial resolution, correspondingly small beams are necessary. While super-resolution techniques such as ptychography show details smaller than the beam size, they have not been applied to energies above $30 \mathrm{keV}$. Also, focussing of X-rays above $30 \mathrm{keV}$ is today only possible using compound refractive lenses (CRLs), that achieve few $\mu \mathrm{m}$ spot sizes at these energies. Fresnel Zone Plates, known from soft X-ray optics [Schmahl], have been generalised to Multilayer Zone Plates and Multilayer Laue Lenses, that can be fabricated with an optical thickness of many $\mu \mathrm{m}$, and hence are promising candidates for efficient focusing of $\geq 60 \mathrm{keV}$ to spot sizes below $100 \mathrm{~nm}$ [1-7].

Here, we demonstrate for the first time sub-50 $\mathrm{nm}$ real-space resolution in a nano-WAXS experiment at an X-ray energy at $60 \mathrm{keV}$ and above $100 \mathrm{keV}$. 


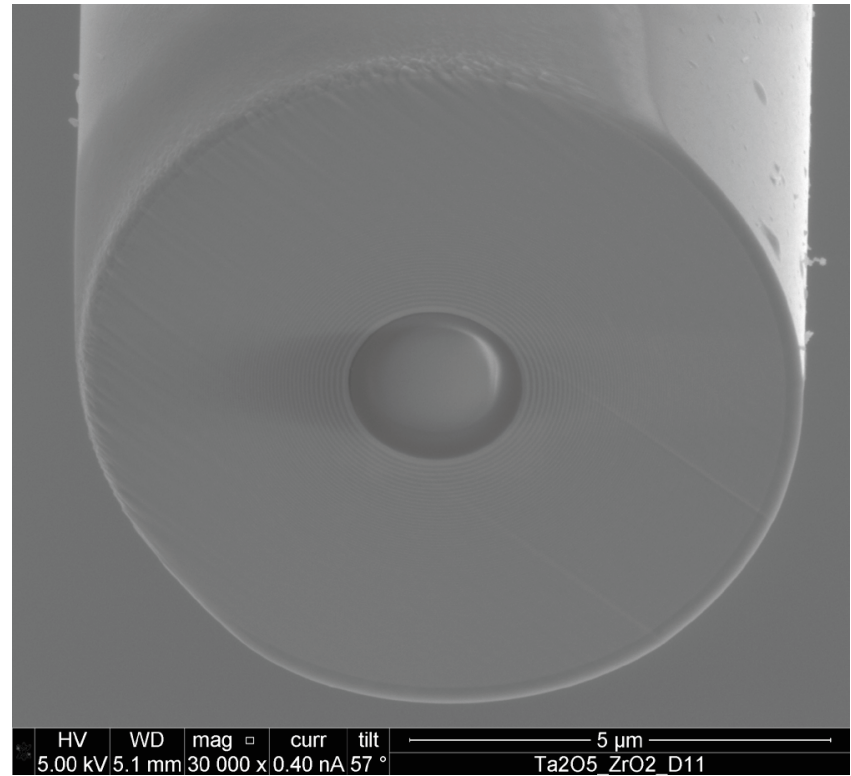

Figure 1a. Front SEM view of the high- $E$ MZP, showing an $8 \mu \mathrm{m}$ aperture. Note that the distortions stem from the charged glass fibre and are not real.

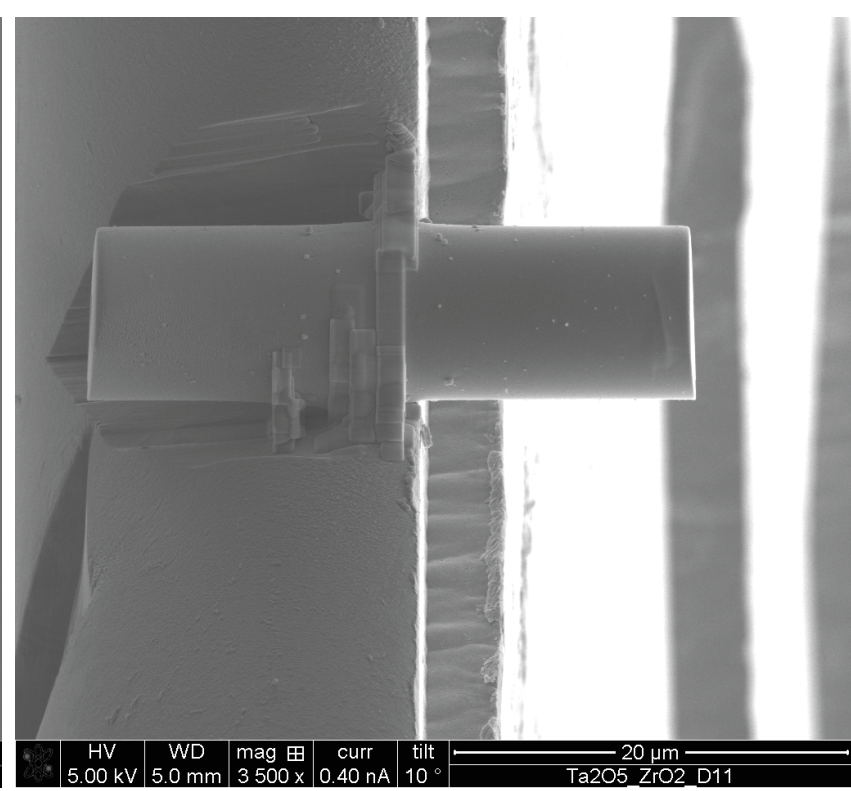

Figure 1b. Side SEM view of the high- $E$ MZP, showing the optical thickness of $30 \mu \mathrm{m}$; the length is designed for optimum phase shift at $E=60 \mathrm{keV}$, assuming an ideal lens.

\section{FABRICATION}

Here we briefly describe the fabrication of MZPs using pulsed laser deposition (PLD) and focused ion beam (FIB); for more details, see [5].

\section{Glass Fibres}

The MZP is deposited onto a rotating glass fibre as a substrate. These fibres are prepared in a heat-and-pull process of standard glass fibres, using a Sutter instruments flaming/brown micropipette puller system P1000 [8]. Changing several parameters (e.g. temperature conditions, heating times and pulling force, to name a few), both the diameter and the opening angle can be adjusted to the needs depending on the MZP design. An MZP with a tapering angle along the optical axis approximates a tilted or wedged design, and can improve the focusing efficiency into the $+1^{\text {st }}$ order.

We note that these glass fibres are millimetres long, and the usable region easily exceeds hundreds of micro metres. With a homogeneous deposition, the concerns regarding optical thickness or aspect ratio known from lithography do not apply for these MZPs.

\section{Pulsed Laser Deposition}

For this particular experiment, a glass fibre has been drawn to a mean thickness of $2.2 \mu \mathrm{m}$, and 191 layers starting from $n=15$ to $n=206$ have been deposited according to the zone plate law. Designed for a focal length of $f=4 \mathrm{~mm}$ and an $\mathrm{x}$-ray energy of $E=60 \mathrm{keV}$, the outermost zone width is thus $10.0 \mathrm{~nm}$ with an overall aperture of $8.2 \mu \mathrm{m}$. The tapering angle of the glass fibre is approximately $2.4 \mathrm{mrad}$.

Although the PLD targets are $\mathrm{Ta}_{2} \mathrm{O}_{5}$ and $\mathrm{ZrO}_{2}$, we have shown that the deposited stoichiometry is given as $\mathrm{Ta}_{2} \mathrm{O}_{1}$ and $\mathrm{ZrO}_{1.8}$; the deviation can be explained by re-sputtering and back-scattering; by this, the ratio between phase shift and absorption and hence efficiency is increased. We note that the stoichiometry on a fibre is even different from a planar substrate. The precise composition is crucial to calculate the indices of refraction determining the optical thickness [5].

\section{Focused Ion Beam}

After deposition, the coated fibre is inspected with an SEM, and the final MZP can be prepared by FIB slicing and polishing. For the given stoichiometry, the difference in index of refraction is given by $\Delta n \approx 1.2 \times 10^{-3} /(E / \mathrm{keV})^{2}$. For a phase shifting zone plate, the optical thickness is then given as $t=0.5 \mu \mathrm{m} /(E / \mathrm{keV})$, hence $30 \mu \mathrm{m}$ for the design energy of $60 \mathrm{keV}$. 


\section{SAMPLE}

We have investigated the possibility of MZP-based nano-WAXS measurements: an X-ray beam of high energy is focused to a few nano metre, penetrates deeply into matter, and exciting a Bragg reflection on a localised nano-crystal. As a test sample, Ag droplets (nano crystallites developing during the PLD process for detuned parameters) were buried within $1.5 \mu \mathrm{m}$ thick layers of $\mathrm{ZrO}_{2}$ on a planar $\mathrm{Si}$ substrate. The typical droplet size is on the single $\mu \mathrm{m}$ scale. Fig. 2a shows a top SEM view of the specimen. Although the droplets are buried, their shape and position can be seen as imprints on the surface. The inset shows an SEM image of a Ag droplet exposed with a FIB after the beamtime.

\section{EXPERIMENT}

Beamline ID31 @ ESRF

ID31 is the high-energy beamline to study buried interfaces and materials at the ESRF. The X-ray beam is provided by an in-vacuum cryo-cooled undulator with a $14.5 \mathrm{~mm}$ period, and it is currently the brightest source in the energy range of 20 to $150 \mathrm{keV}$ [9]. The beamline is equipped with three sets of compound refractive lenses; during the described experiment, we have used 33 Be lenses in the first optical hutch, and $61(60 \mathrm{keV})$ to $255(101 \mathrm{keV}) \mathrm{Be}$ lenses in the second optical hutch as a prefocus.

\section{Laue-Laue monochromator}

This was the first user experiment with a new Laue-Laue bending monochromator. Two $\mathrm{Si}(111)$ crystals in non-dispersive geometry can be rotated to select the desired energy; in addition, the energy bandwidth can be selected with a new bender developed at the ESRF [9]. For this work, a bandwidth of $0.44 \%$ was chosen at $60 \mathrm{keV}$, and $0.57 \%$ at $101 \mathrm{keV}$. The undulator was operated on the $5^{\text {th }}$ and $9^{\text {th }}$ harmonic, respectively.

\section{Detector: Pilatus3 CdTe 2M}

We are glad that we could use the new Pilatus detector (Dectris Inc., Baden, Switzerland) with a CdTe sensor chip, allowing for high quantum efficiency at $60 \mathrm{keV}$ and still about $50 \%$ at $100 \mathrm{keV}$. During the beamtime, the detector was translated between $0.86 \mathrm{~m}$ and $6.0 \mathrm{~m}$ downstream the MZP, so it could both be used as a WAXS detector for the Ag nano crystal signals as well as for alignment of the MZP. Unfortunately, the pixel size is too large (at this energy and distance) for ptychography that could yield quantitative information about the focal spot size.

\section{Setup: HEMD}

The MZP-sample tower has been integrated into the HEMD diffractometer of ID31, see Fig. 2b for a photo.

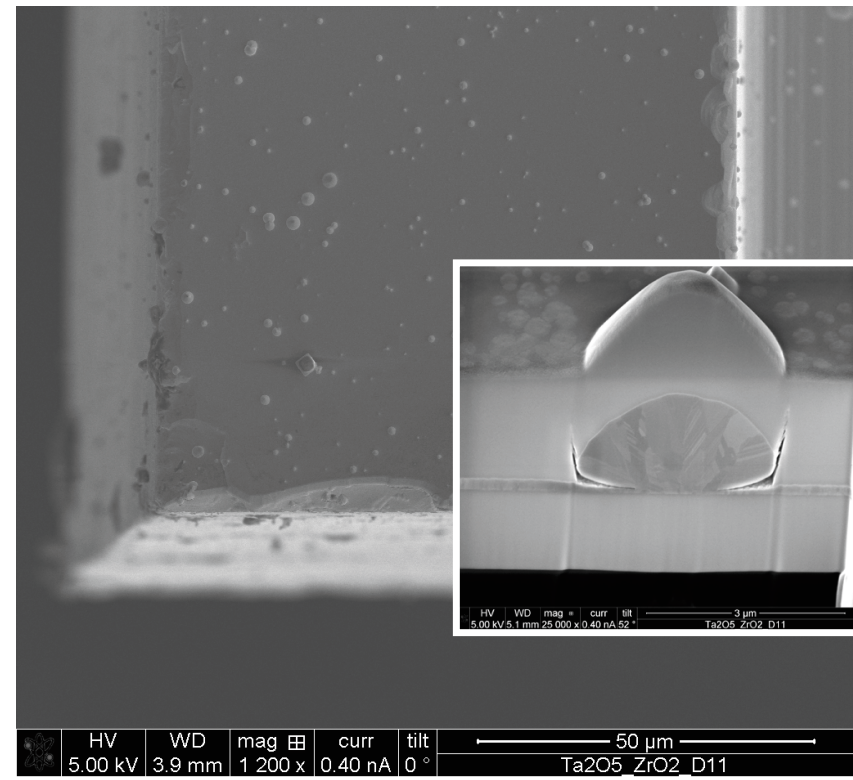

Figure 2a. An imprint of Ag droplets / nanocrystals, sandwiched between two layers of $\mathrm{ZrO}_{2}$ (SEM top view). The inset shows an SEM image of one droplet after it has been exposed with FIB.

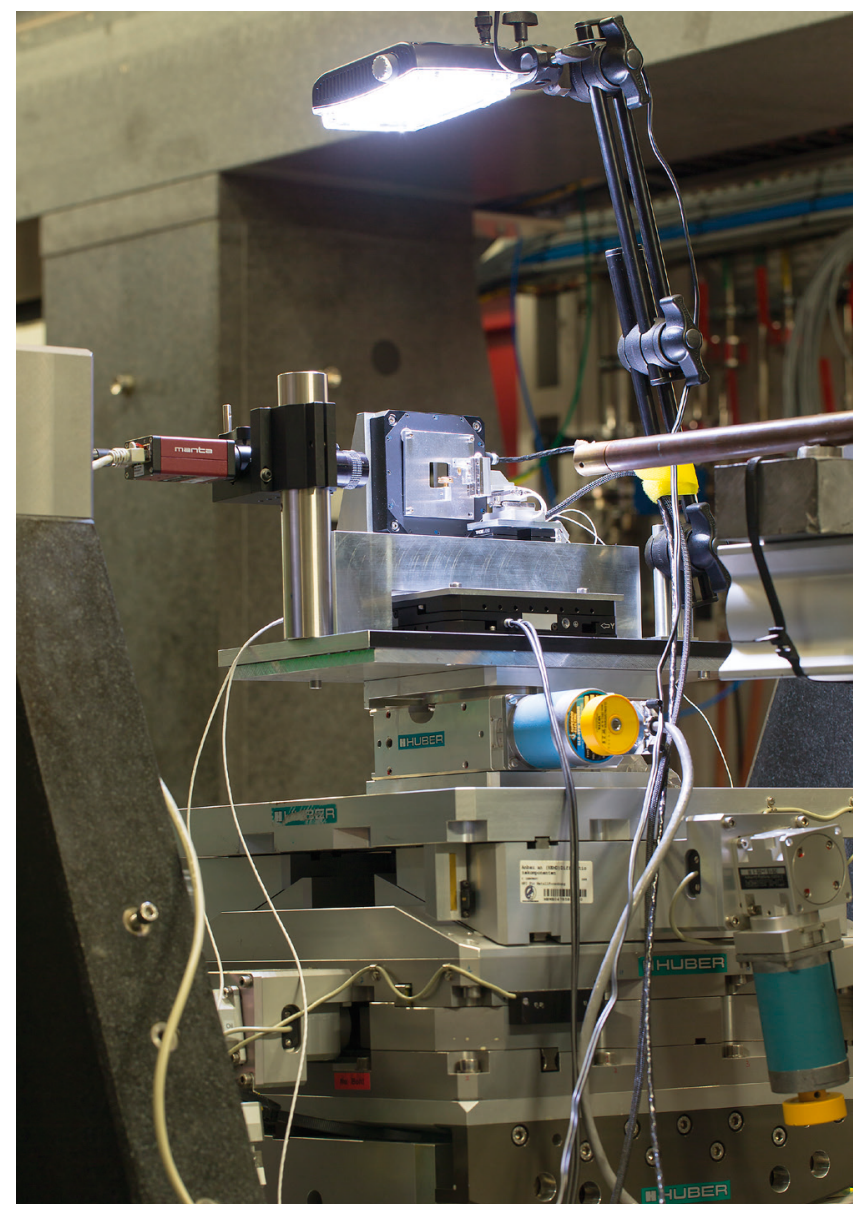

Figure 2b. MZP-sample tower integrated into the HEMD diffractometer at the high-energy beamline ID31 of the ESRF. 


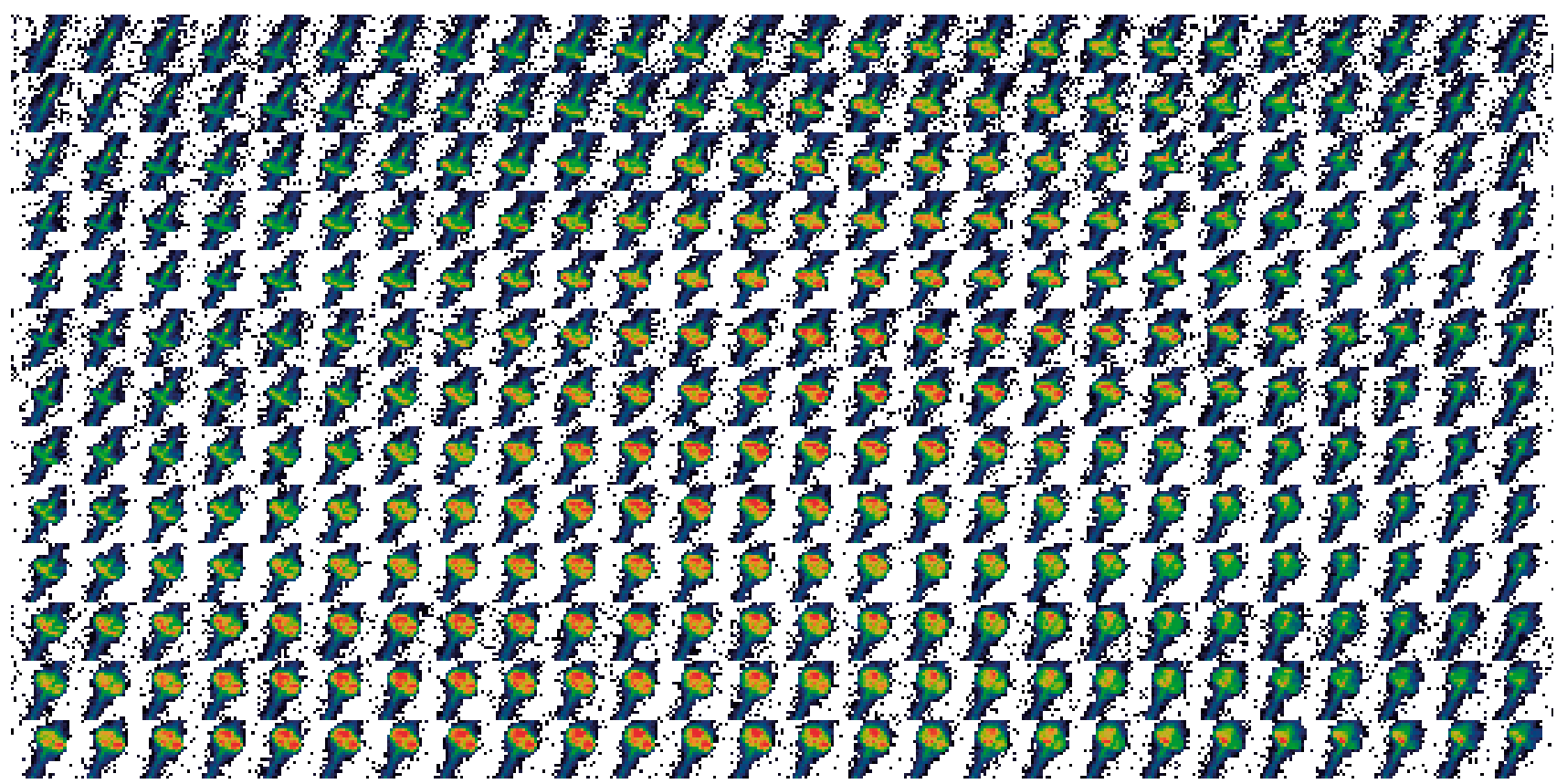

Figure 3. Demonstration of nano-WAXS with high X-ray energies: The Ag droplet was raster scanned in the MZP focal plane (field of view in real space shown here: $520 \mathrm{~nm} \times 600 \mathrm{~nm}$ ), at its Bragg peak is recorded with the Pilatus detector in $860 \mathrm{~mm}$ distance. The intensity pattern forms due to a convolution of the droplet's strain with the MZP illumination.

\section{Motivation}

\section{SCANNING NANO-WAXS}

A high-energy scanning nano beam enables to very locally excite Bragg peaks of nano crystallites buried deeply inside matter (which can be amorphous either or crystalline with a different layer spacing). At high X-ray energies, the Ewald sphere becomes more and more flat, and reciprocal space contracts. Larger parts of $q$-space are hence accessible in shorter time, and (in real-world diffractometer setups) with fewer rotations. When combined with fluorescence, more K-shells can be probed.

\section{Experimental parameters}

We have used an MZP fabricated with an outer diameter of $8 \mu \mathrm{m}$, an optical thickness of $30 \mu \mathrm{m}$ and outermost layers of $10 \mathrm{~nm}$; for the experiments, the MZP has been aligned in a CRL prefocus of about $20 \mu \mathrm{m}$. The $0^{\text {th }}$ order MZP beam (unfocused beam) has then be used to find crystals within the bulk sample. The MZP has been centred inside the CRL beam, and could be moved in and out within a few seconds, enabling a fast switching of pre-focus for low-resolution alignment and high-resolution experiments. The X-ray energy has been set to first $60 \mathrm{keV}$, and in the last day to $101 \mathrm{keV}$. An MZP with an optical thickness of $50 \mu \mathrm{m}$ or more - needed for higher efficiency at the final energy - would be possible to fabricate.

\section{Nano-WAXS scans}

One typical example of a nano-WAXS scan is shown in Fig. 3. Depicted is the ROI on the Pilatus CdTe detector of the signal of one individual Ag droplet (small images), laid out in a 2D grid corresponding to the scanning positions of the sample in the X-ray beam. At the top edge and corners a green stripe is visible, which is attributed to the homogeneous background of the CRL beam (which is of rather infinite size in the given field of view of only $0.5 \mu \mathrm{m} \times 0.6 \mu \mathrm{m}$ ).

In the central region of the scan, the Bragg peak is excited by the MZP beam. The signal is increased by a factor of about three compared to the CRL beam, and a variety of sub-structures become visible. During this very first beamtime joining MZP nano-focus and high X-ray energies, the sample has not been aligned very well in the focal plane; therefore, the diffraction patterns show the convolution of the MZP focus' phase front with the internal lattice distortions of the Ag droplet. With careful alignment, these distortions caused by stress and strain during droplet formation and after deposition could be analysed in more detail and quantitatively. 


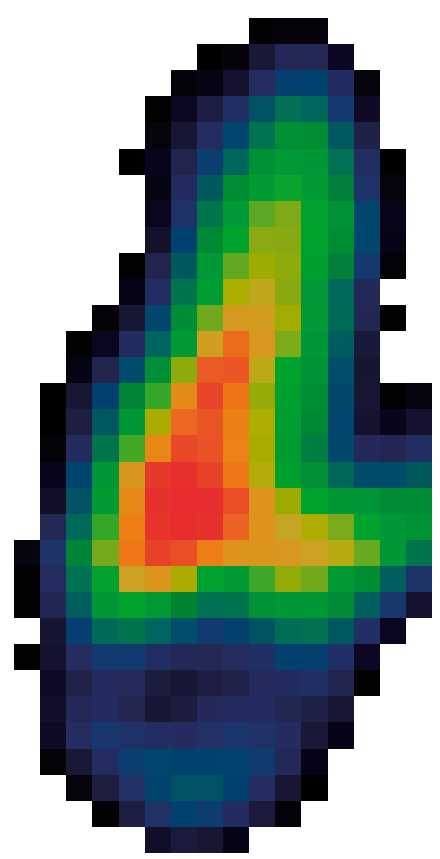

Figure 4a. Spatially resolved Bragg peak intensity, field of view $0.8 \mu \mathrm{m} \times 1.6 \mu \mathrm{m}$

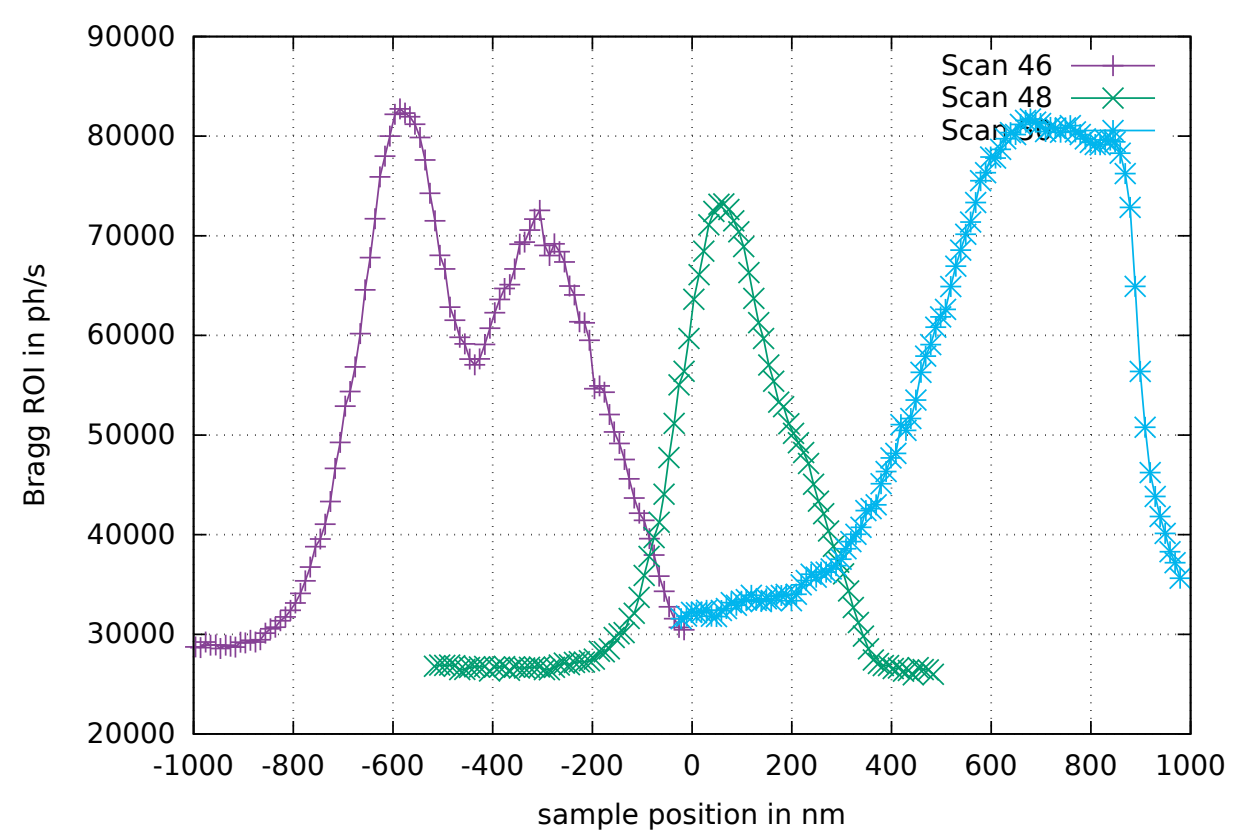

Figure 4b. 1D line scans of the same droplet (shifted for clarity), showing substructure in scan 46 (purple). In case of scan 50 (light blue), the droplet serves as a knife edge with a steepness of better than $50 \mathrm{~nm}$. Note that the droplet was not actually in the best focal plane of the MZP.

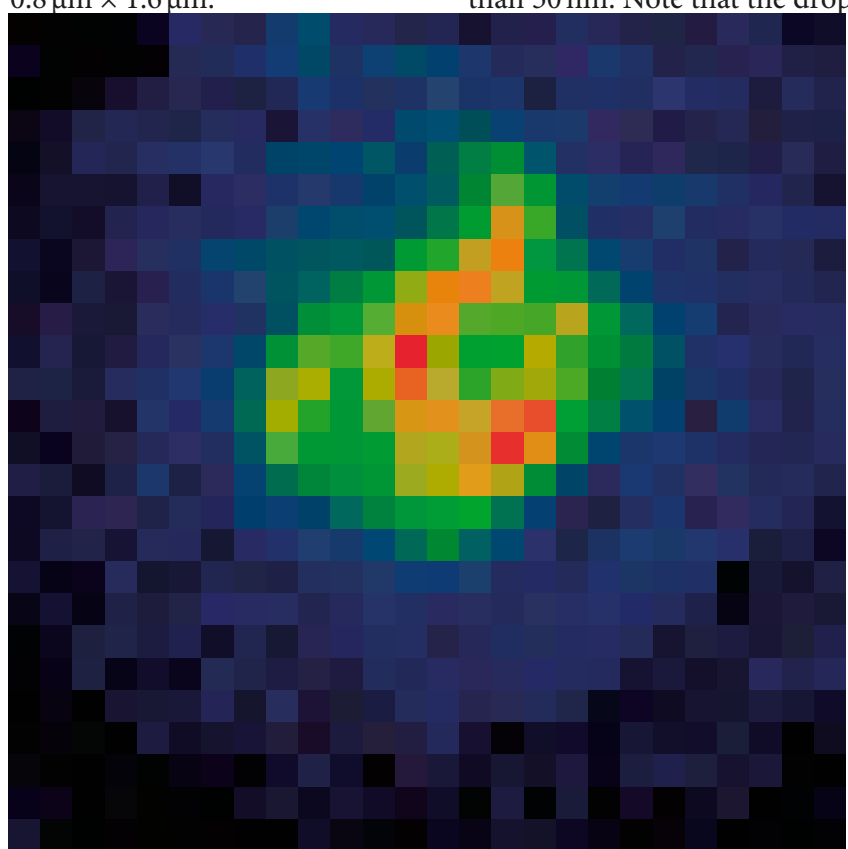

Figure 5a. Spatially resolved Bragg peak intensity @ $101 \mathrm{keV}$, field of view $2 \mu \mathrm{m} \times 2 \mu \mathrm{m}$. Note: this is a different droplet than in Fig. 4 .

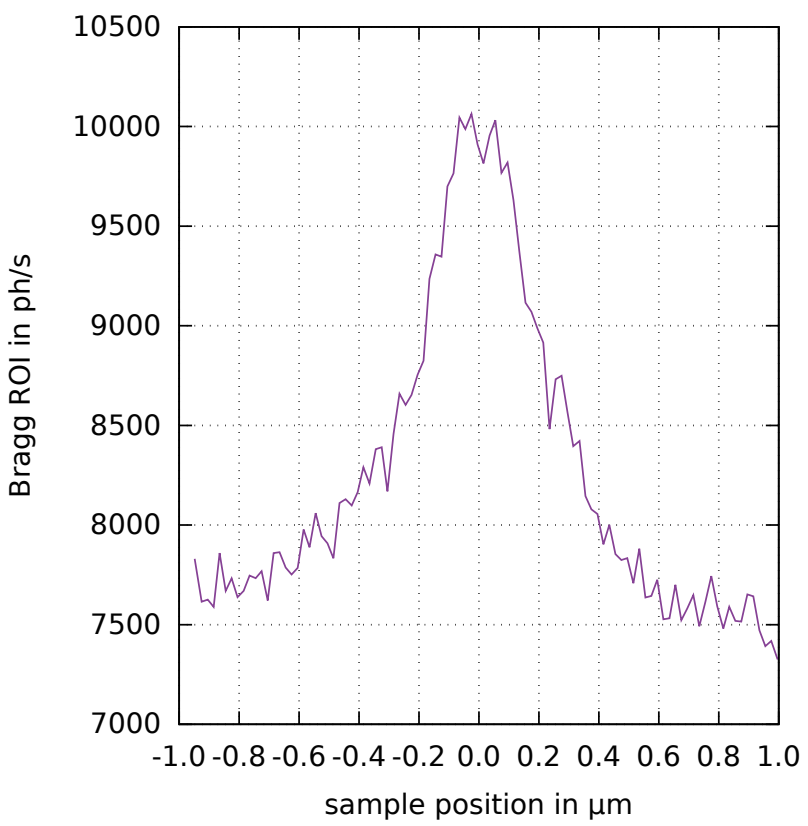

Figure 5b. 1D line scan of the same droplet as in Fig. 5a; the steepness is better than $40 \mathrm{~nm}$ at an X-ray energy of $101 \mathrm{keV}$.

\section{Ag droplet as knife edge}

The nano crystallites serve as a dark-field knife edge to quantify the X-ray focal spot size, as presented in Fig. 4 and Fig. 5 for the X-ray energies of $60 \mathrm{keV}$ and $101 \mathrm{keV}$, respectively. The integrated Bragg peak intensity of the detector ROI is shown as a 2D map of the scanning position in Fig. 4a and Fig. 5a; the images correspond to the real-space extent of a single crystalline domain of the Ag droplet buried inside $\mathrm{ZrO} 2$. In Figs. $4 \mathrm{~b}$ and 5b, 1D line cuts of the 2D images with a steepness of down to $50 \mathrm{~nm}(60 \mathrm{keV})$ and $40 \mathrm{~nm}(101 \mathrm{keV})$ serve as estimated of the focal spot size. 


\section{CONCLUSIONS \& OUTLOOK}

We have shown that the combination of compound refractive lenses as stable and well-established pre-focusing optics for high X-ray energies with high-resolution multilayer zone plates allows to probe and spatially resolve an individual domain of Ag nano crystals. From 1D dark-field scans in a nano-WAXS setup, the resolution has been determined to be better than $50 \mathrm{~nm}$ at $60 \mathrm{keV}$ and even better than $40 \mathrm{~nm}$ at $101 \mathrm{keV}$. The full potential of the MZP focus depends of course on properly aligning the sample in the focal plane, which has not been achieved in the presented experiment. Nonetheless, the results show the feasibility of nano-focussing three-digit energies that are able to penetrate into nano-sized particles buried deeply inside bulk material.

Other geometries can also be thought of, e.g. X-ray reflectivity at deeply buried interfaces to detect very localised defects; using fluorescence detectors, Compton scattering and fluorescence data can be used to gain insights into chemical compositions and physical structures in-situ, which are not be accessible with other techniques. The combination of high X-ray energies with penetration lengths of millimetres even in metals, with a nano-focusing optic, can open new ways of materials and structure investigation.

\section{REFERENCES \& ACKNOWLEDGEMENTS}

[1] H.C. Kang, H. Yan, R.P. Winarski, M.V. Holt, J. Maser, C. Liu, R. Conley, S. Vogt, A.T. Macrander, G.B. Stephenson: Focusing of hard X-rays to 16 nanometers with a multilayer Laue lens, Applied Physics Letters 92, 221114 (2008).

[2] H. Mimura, S. Handa, T. Kimura, H. Yumoto, D. Yamakawa, H. Yokoyama, S. Matsuyama, K. Inagaki, K. Yamamura, Y. Sano, J. Tamasaku, Y. Nishino, M. Yabashi, T. Ishikawa, K. Yamauchi: Breaking the $10 \mathrm{~nm}$ barrier in hard-X-ray focusing, Nature Physics 6, 122-125 (2010).

[3] X. Huang, H. Yan, E. Nazaretski, R. Conley, N. Bouet, J. Yhou, K. Lauer, L. Li, D. Eom, D. Legnini, R. Harder, I.K. Robinson, Y.S. Chu: $11 \mathrm{~nm}$ hard X-ray focus from a large-aperture multilayer Laue lens, Scientific Reports 3, 3562 (2013).

[4] F. Döring, A.L. Robisch, C. Eberl, M. Osterhoff, A. Ruhlandt, T. Liese, F. Schlenkrich, S. Hoffmann, M. Bartels, T. Salditt, H.U Krebs: Sub-5 $\mathrm{nm}$ hard x-ray point focusing by a combined Kirkpatrick-Baez mirror and multilayer zone plate, Optics Express 21, 19311-19323 (2013).

[5] C. Eberl, F. Döring, T. Liese, F. Schlenkrich, B. Roos, M. Hahn, T. Hoinkes, A. Rauschenbeutel, M. Osterhoff, T. Salditt, H.U. Krebs: Fabrication of laser deposited high-quality multilayer zone plates for hard x-ray nanofocusing, Applied Surface Science 307 638-644 (2014).

[6] J.C. da Silva, A. Pacureanu, Y. Yang, S. Bohic, C. Morawe, R. Barrett, P. Cloetens: Efficient concentration of high-energy x-rays for diffractin-limited imaging resolution, Optica 4, 492-495 (2017).

[7] S. Bajt, M. Prasciolu, A.J. Morgan, H.N. Chapman, J. Krzywinski, A. Andrejczuk: One dimensional focusing with high numerical aperture multilayer Laue lens, AIP Conference Proceedings 1696, 020049 (2016).

[8] F. Warken, A. Rauschenbeutel, T. Bartholomäus: Fiber pulling profits from precise positioning, Photon. Spectra 42, 73-75, 2008.

[9] M. Magnin-Mattenet, P. Got, A. Vivo, V. Honkimäki: A new crystal bender for the ID31 Laue-Laue monochromator, Proc. MEDSI2016 (2016).

\section{Funding}

We acknowledge funding by Deutsche Forschungsgemeinschaft through SFB 755 "Nanoscale Photonic Imaging"; we thank the ESRF for granting beamtime and Veijo Honkimäki and his team for excellent support during the experiment. 